\title{
Metabolic profile of chronic liver disease by NMR spectroscopy of human biopsies
}

\author{
BEATRIZ MARTÍNEZ-GRANADOS ${ }^{1}$, JOSE MANUEL MORALES ${ }^{2}$, JOSÉ MANUEL RODRIGO ${ }^{3}$, \\ JUAN DEL OLMO ${ }^{3}$, MIGUEL ANGEL SERRA ${ }^{3}$, ANTONIO FERRÁNDEZ ${ }^{4}$, \\ BERNARDO CELDA $^{1,5}$ and DANIEL MONLEÓN ${ }^{6}$
}

\author{
${ }^{1}$ Departamento de Química Física, ${ }^{2}$ Unidad central de Investigación en Medicina, ${ }^{3}$ Servicio de Hepatología, Hospital \\ Clínico Universitário de Valencia, Departamento de Medicina, Universidad de Valencia; ${ }^{4}$ Servicio de Anatomia \\ Patológica, Hospital Clínico de Valencia; ${ }^{5}$ CIBER Bioengineering, Biomaterials and Nanomedicie, ISC III; \\ ${ }^{6}$ Fundación de Investigación del Hospital Clínico Universitario de Valencia, INCLIVA, Valencia, Spain
}

Received July 27, 2010; Accepted September 13, 2010

DOI: $10.3892 / \mathrm{ijmm} .2010 .563$

\begin{abstract}
Among the different processes occurring during the evolution of liver disease, fibrosis has a predominant role. Liver fibrosis mechanisms are fairly constant irrespective of the underlying etiology. Cirrhosis is the end-stage of this reaction. Metabolic profiles, which are affected by many physiological and pathological processes, may provide further insight into the metabolic consequences of this severe liver disease. The aim of this study was to demonstrate the applicability of ${ }^{1} \mathrm{H}$ high resolution magic angle spinning (HR-MAS) NMR spectroscopy in the biochemical profile determination of human liver needle biopsy samples for the characterization of metabolic alterations related to the severity of liver disease. We recorded and analyzed HR-MAS spectra of 68 liver tissue samples obtained by needle biopsy from patients with chronic liver disease. Multivariate analysis was applied to these data to obtain discrimination patterns and to reveal relevant metabolites. The metabolic characterization of liver tissue from needle biopsies by HR-MAS NMR spectroscopy provided differential patterns for cirrhotic and non-cirrhotic chronic liver disease tissue. Metabolites closely related to the liver metabolism such as some fatty acids, glucose and amino acids show differences between the two
\end{abstract}

Correspondence to: Dr Daniel Monleón, Fundación de Investigación del Hospital Clínico Universitario de Valencia, INCLIVA, Avda. Blasco Ibáñez 17, Valencia 46010, Spain E-mail: daniel.monleon@uv.es

Abbreviations: HR-MAS, high resolution magic angle spinning; TOCSY, ${ }^{1} \mathrm{H}-{ }^{1} \mathrm{H}$ total correlation spectroscopy; CPMG, Carr-PurcellMeiboom-Gill; NOESY, nuclear Overhauser effect spectroscopy; HSQC, heteronuclear single quantum coherence spectroscopy

Key words: metabolomics, metabolic profiling, NMR spectroscopy, chronic liver disease, HR-MAS, liver needle biopsy groups. Phospholipid precursors, which have been previously correlated with hepatic lesions also show differences. Furthermore, the correlation between histologically assessed liver disease stages and the levels of the most discriminative metabolites show that liver dysfunction is present at the initial stages of chronic hepatic lesions. Overall, this work suggests that the additional information obtained by NMR metabolomics applied to needle biopsies of human liver may be useful for assessing metabolic alterations and liver dysfunction in chronic liver disease.

\section{Introduction}

The liver is among the most metabolically diverse organs of the body and is involved in many critical metabolic processes (1). One of its major roles is storing endogenous fuels of the body. In addition, the liver acts as a major distribution center for exogenous energy supplies. The liver may be affected by many pathological aggressions. Liver dysfunction results in a variety of clinical signs and symptoms. However, some of these pathologies pose a diagnostic challenge as many different diseases show similar clinical signs (2-4). Moreover, liver biopsy assessment involves some degree of complication because of the proliferation of different grading and staging schemes (5-7). Metabolic alterations produced by liver disease may help clinicians better characterize the disease. Among the different processes occurring during the evolution of liver disease, fibrosis takes a predominant role. Liver fibrosis is characterized by the replacement of liver tissue by fibrous scar tissue as well as regenerative nodules, leading to progressive loss of liver function and to altered liver metabolism (8-11). Liver fibrosis induction mechanisms are fairly constant irrespectively of the underlying etiology (12-16). Cirrhosis is the end-stage of this reaction and it represents a major change in the tissue. Most of the conventional approaches for the assessment of liver disease rely on few biomarkers, which represent particular disease states and often are not sufficient for providing global biochemical characterization and definitive diagnosis (17-20). Global metabolic profiles, which are affected by many 
physiological and pathological processes, may more accurately reflect the presence of a particular disease state. Recent studies of NMR lipid profiling in chronic hepatitis C show the potential of this approach (21-24). The high metabolic activity of the liver makes the hepatic disease a perfect candidate for metabolic profiling.

High resolution magic angle spinning (HR-MAS) NMR spectroscopy is a powerful technique for the investigation of metabolites within different intact tissues (25-31). The precise determination of the biochemical and metabolic profiles in intact tissue promises to extend the possibilities of NMR as a medical diagnostic tool. For non-solid or highly viscous liquids, HR-MAS NMR spectroscopy allows the reduction of most of the line broadening associated with restricted molecular motion, chemical shift anisotropy, dipolar couplings and field heterogeneity, by high-rate spinning of the sample at the magic angle $\theta=54.7^{\circ}(32)$. The potential of HR-MAS applications to the study of biological tissues has been widely demonstrated in the investigation of different cellular alterations $(30,33)$. In addition, HR-MAS NMR spectroscopy of intact tissues (ex vivo) provides further advantages over traditional high resolution liquid NMR of tissue extracts (in vitro) $(27,34)$. High resolution NMR of extracts from excised tissues requires large amounts of sample (35). Likewise, the extraction process via protein precipitation methods disables the direct observation of small cellular proteins and membrane semi-mobile lipids. Moreover, extraction methods usually discriminate metabolites on the basis of their solubility in particular solvents. Although it has some minor limitations associated mainly to the spinning of the sample (spinning side-bands, spinning degradation effects and spinning temperature gradients among others), HR-MAS NMR is a non-destructive technique, which requires minimal sample preparation, and allows the observation of most of the tissue metabolites and dynamic interactions in an extremely reduced sample quantity. This technology can supplement histopathological examination and improve liver disease diagnostics $(21,28,36,37)$. The similarities between 'ex vivo' and 'in vivo' spectra found in studies of different tumours allow better interpretation of in vivo MR spectra and increase the clinical potential of the method (38). HR-MAS spectra generate metabolic profiles that contain information on the physiological and pathological status. This approach can be used to define the metabolomic phenotype of a tissue. Previous studies have shown the potential of HR-MAS NMR spectroscopy as a tool for liver disease characterization $(21,28,36)$. Furthermore, studies in human liver tissue suggested the applicability of the technique in the assessment of liver transplant viability (36).

The aim of this study was to demonstrate the applicability of ${ }^{1} \mathrm{H}$ HR-MAS NMR spectroscopy in the biochemical profile determination of human liver needle biopsy samples for the assessment of the chronic liver disease stage.

\section{Patients and methods}

Patients and tissue collection procedure. Sixty-eight samples of liver tissue were obtained from 68 chronic liver disease patients at the Service of Hepatology of the Hospital Clinico Universitario of Valencia. The biopsy tissue was collected by
Table I. Summary of patient and sample information for this study.

\begin{tabular}{lccccc}
\hline & \multicolumn{3}{c}{ Chronic hepatitis } & & Cirrhosis \\
\cline { 2 - 4 } \cline { 5 - 6 } Disease stage & 1 & 2 & 3 & \\
\hline Etiology & 12 & 20 & 14 & & 6 \\
$\quad$ Virus C & - & 1 & 1 & & 1 \\
Alcohol & - & - & - & & 1 \\
Virus C + Alcohol & - & - & - & \\
Cryptogenic & 2 & 1 & 2 & \\
Autoimmune & 2 & 2 & - & \\
Virus B & $36-60$ & $35-66$ & $32-68$ & & $38-76$ \\
Age range (years) & $9 / 7$ & $18 / 6$ & $10 / 7$ & $6 / 5$ \\
Gender (M/F) & & & & \\
\hline
\end{tabular}

an anatomopathologist for routine histological analysis and the informed consent was obtained from all patients. The study was approved by the local Ethics Committee. The remainder tissue was immediately put in cryogenic vials and snap-frozen in liquid nitrogen. All samples used for histopathological examination were fixed in formalin and paraffin embedded by a pathologist. Routine methods were employed for histopathological studies. All snap-frozen samples for HR-MAS were at $-80^{\circ} \mathrm{C}$ until further analysis. Liver tissue analyzed included 11 cirrhotic and 57 noncirrhotic samples obtained from 43 male and 25 female patients, 32-76 years of age. Histopathological grade and stage was assessed according to the Batts-Ludwig classification (39). Table I gives a summary of the clinical characterization of the patients and the biopsy samples.

Sample preparation. Liver tissue obtained for HR-MAS measurements was stored at $-80^{\circ} \mathrm{C}$ until use. Due to the small size of the samples (approximately 1-12 mg), all the materials that were to be in contact with the tissue were cooled to at least $4^{\circ} \mathrm{C}$ to reduce tissue degradation during the sample preparation process. Frozen samples were placed in a cryovial and in liquid $\mathrm{N}_{2}$ until insertion in a 4-mm outer diameter $\mathrm{ZrO}_{2}$ rotor. The whole operation took no longer than $2 \mathrm{~min}$. The pre-cooled rotor was filled with $4^{\circ} \mathrm{C}$ cooled $\mathrm{D}_{2} \mathrm{O}$ after sample insertion. Spherical inserts were used in all the cases, limiting the rotor inner volume to $12 \mu 1$. HR-MAS using spherical small volume inserts provides improved resolution and line shapes compared to cylindrical inserts (40). $\mathrm{D}_{2} \mathrm{O}$ exceeding the $12 \mu \mathrm{l}$ volume was removed before rotor sealing. Due to the small amount of tissue and the possible degradation during the sample weighting process, tissue samples were weighted only after HR-MAS measurements.

${ }^{1} H$ HR-MAS NMR spectroscopy of intact human liver tissue. All spectra were recorded in a Bruker Avance DRX 500 spectrometer (Valencia, Spain) operating at a ${ }^{1} \mathrm{H}$ frequency of $500.13 \mathrm{MHz}$. The instrument was equipped with a $4 \mathrm{~mm}$ 
triple resonance ${ }^{1} \mathrm{H} /{ }^{31} \mathrm{P} /{ }^{13} \mathrm{C} \mathrm{HR}-\mathrm{MAS}$ probe. For all experiments, samples were spun at $4000 \mathrm{~Hz}$ to keep the rotation sidebands out of the acquisition window. Field homogeneity, required for adequate resonances, line-shape and spectral resolution, was achieved by extensive coil shimming using the 1D water pre-saturation experiments in the interactive mode as a control. When detected, the lactate doublet at $1.33 \mathrm{ppm}$ was used for field homogeneity shimming, as described elsewhere (41). Sample temperature, as calibrated using $\mathrm{MeOH}$ standards (38), was kept at $4^{\circ} \mathrm{C}$, using the cooling of the inlet gas pressures responsible for the sample spinning. The number of transients varied from 400 to 2048 depending on the tissue sample amount. Water pre-saturation was used during the last sec of the recycling delay in all experiments for solvent signal suppression. Experiments acquired for all samples always included 1D pre-saturation ${ }^{1} \mathrm{H}$ spectra and $\mathrm{T}_{2}$ edited 1D Carr-PurcellMeiboom-Gill (CPMG) ${ }^{1} \mathrm{H}$ spectra (echo time of $400 \mathrm{msec}$ ) in order to minimize the contribution of macromolecules to the profile. However, CPMG spectra did not provide enough signal-to-noise ratios for subsequent analysis (data not shown). 2D experiments were acquired on selected samples for assignment purposes (Fig. 2). Recycling delays were $1.5 \mathrm{sec}$ for all experiments. Chemical shift referencing was performed relative to a cluster of fatty acid signals, which was detected in all experiments.

Spectral analysis. All 68 spectra were processed using TopSpin 1.5 (Bruker BioSpin GmbH, Rheinstetten, Germany) and transferred to MATLAB (MathWorks Inc., 2006) using in-house scripts for data analysis. The chemical shift region including resonances between 2.00 and $4.40 \mathrm{ppm}$ (the aliphatic region) was investigated. The aromatic region did not provide enough signal-to-noise for the subsequent analysis. The spectra were normalized to the aliphatic spectral area between 2.00 and $4.40 \mathrm{ppm}$ containing most intracellular metabolite signals for avoidance of major fatty acid signal and to eliminate differences in relative fat content. The spectra were binned into $0.01 \mathrm{ppm}$ buckets and statistical analysis was performed using in-house MATLAB scripts and the PLS_Toolbox statistical multivariate analysis library. A high variability among the size, weight and fat content of the samples made it necessary to use variance stabilization procedures. The bucketed data were then analyzed by the Partial Least Squares Discriminant Analysis (PLS-DA) for detecting discriminating patterns and metabolites. The PLS-DA model was validated by the leaveone-out method. The averaged loading plots of the corresponding PLS-DA latent variables were used to detect the most relevant metabolites. Signals belonging to these selected metabolites were integrated and relative quantities were obtained using semi-automated in-house MATLAB peak-fitting routines over the normalized spectra. A power calculation by the Mann-Whitney $U$ test at the $5 \%$ significance $(\alpha=0.05)$ was used for the determination of the statistical significance of the differences between corresponding integrals in chronic hepatitis and cirrhotic samples. Differences with respect to the etiology were also explored in a similar fashion. Correlation with liver disease stage was explored by Pearson's linear correlation analysis.
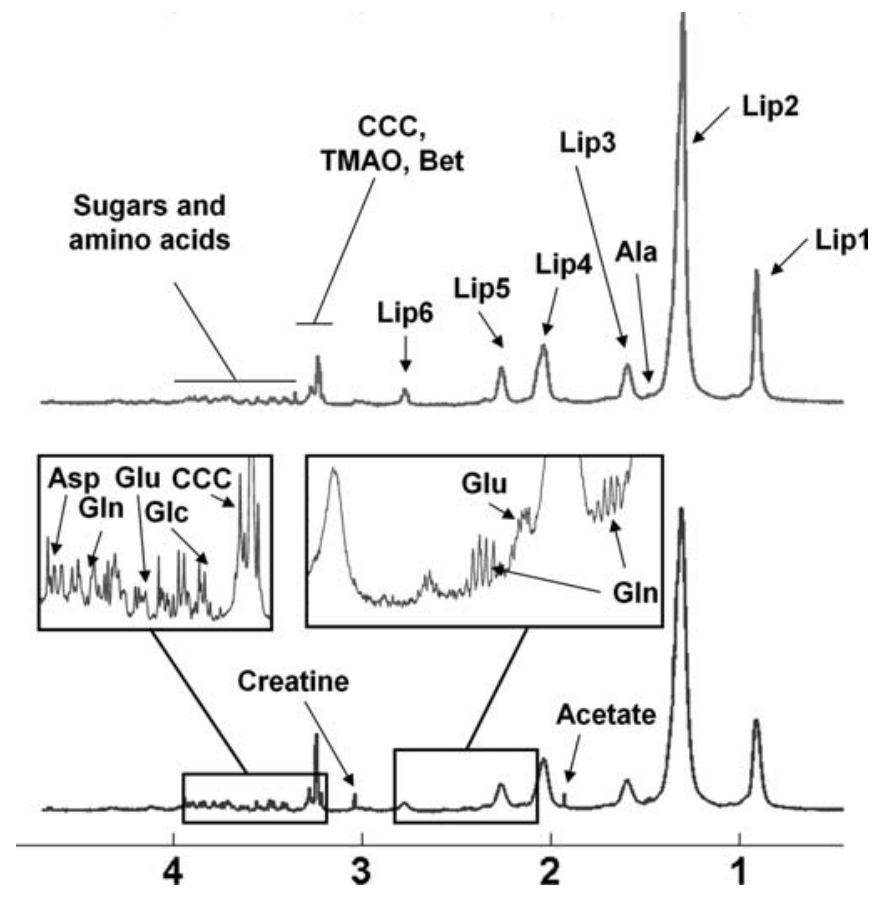

Figure 1. Typical spectra from a ${ }^{1} \mathrm{H}$ HR-MAS $1 \mathrm{D}$ pre-saturation single-pulse experiment for non cirrhotic (bottom) and cirrhotic (top) chronic liver disease tissue with most relevant resonances labeled. Lip1, lipid $\underline{\mathrm{CH}}_{3}$; Lip2, lipid $\left(\underline{\mathrm{CH}}_{2}\right)$ n; Lip3, lipid $\underline{\mathrm{CH}}_{2} \mathrm{CH}_{2} \mathrm{CO}$; Lip4, lipid $\underline{\mathrm{CH}}_{2} \mathrm{CH}=\mathrm{CH}$; Lip5, lipid $\underline{\mathrm{CH}}_{2} \mathrm{CO}$; Lip6, $\mathrm{CH}=\mathrm{CHCH}_{2}-\mathrm{CH}=\mathrm{CH}$; $\mathrm{CCC}$, choline-containing compounds; TMAO, trimethylamine-N-oxide; Bet, betaine; Ala, alanine; Glu, glutamate; Gln, glutamine.

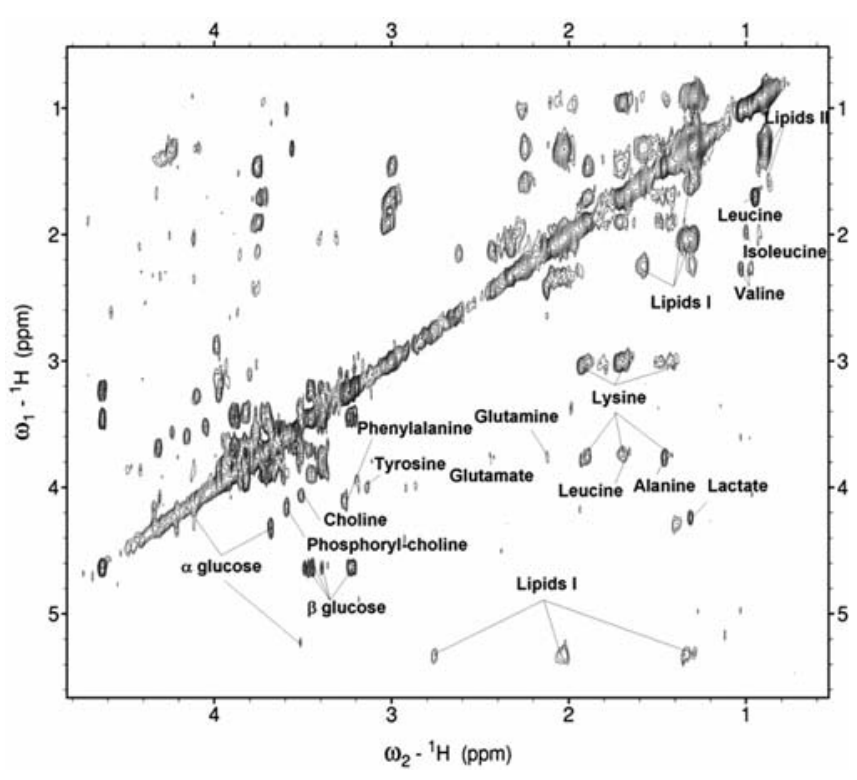

Figure 2. Aliphatic region of 2D 1H,1H TOCSY HR-MAS spectrum with $50 \mathrm{msec}$ of mixing time for $7.5 \mathrm{mg}$ of human liver tissue showing the assignment of the most significant metabolites.

\section{Results}

Spectroscopic data. Representative ${ }^{1} \mathrm{H}$ NMR spectra of cirrhotic and non cirrhotic chronic liver disease tissue are 

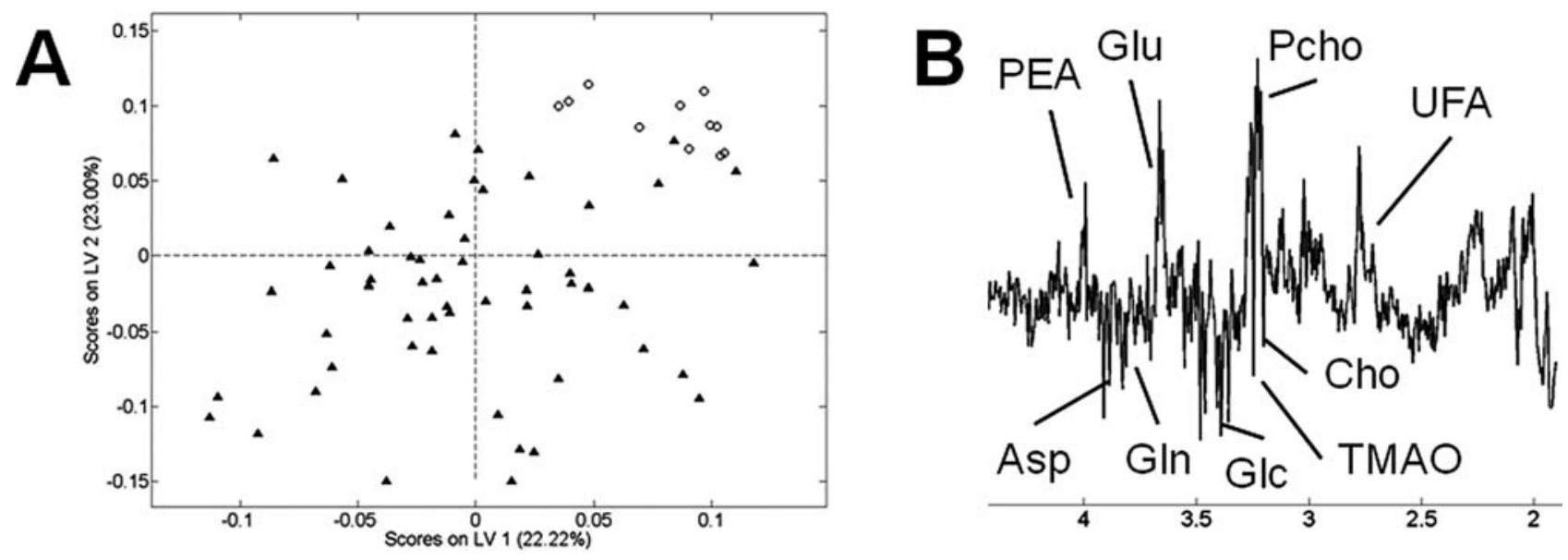

Figure 3. (A). Scores of the LV1 and the LV2 of the PLS-DA model $\left(\mathrm{R}^{2}=30.2 \%, \mathrm{Q}^{2}=78.2 \%\right)$ for the discrimination between cirrhotic (white circles) and noncirrhotic (black triangles) chronic liver disease biopsies based on the ${ }^{1} \mathrm{H}$ HR-MAS 1D presaturation single-pulse experiment. (B). Loadings plot for the PLS-DA model for discrimination between cirrhotic and non cirrhotic chronic liver disease biopsies with labels on the resonances of the most significant metabolites.

shown in Fig. 1. The use of highly symmetric spherical cavity inserts provided high spectral resolution and sensitivity. All NMR spectra were dominated by broad resonances arising from fatty acid mobile chains. Metabolite spin systems and resonances were identified by using literature data $(28,36)$ and were confirmed by additional 2D homo- and multi-nuclear experiments collected in selected samples. The 1D single-pulse pre-saturation experiment provides complete and unambiguous identification of the metabolic pattern characterizing the examined tissues. Collagen, which is a typical molecule present in fibrotic tissue, cannot be observed by conventional NMR techniques due to its size and restricted mobility. The high variability found in terms of the sample size, weight and fat content resulted in very different spectral profiles across the whole data set of samples. In the liver, large molecules are thoroughly present (cholesterol in lipoproteins, lipids, fatty acids, etc) and, although not observable as sharp NMR signals, they may partially distort the spectral baseline. The broad signals belonging to the mobile parts of fatty acids and lipids extensively overlapped with the sharp signals from the lower molecular weight metabolites. In those samples with a lower relative fat content, the decreased overlapping allowed the detection of prominent signals from water-soluble metabolites, such as lactate, glucose, creatine, taurine, phosphoethanolamine, glycerophosphocholine, phosphocholine, choline and most of the amino acids in the aliphatic region (Fig. 1).

Multivariate analysis. HR-MAS spectra from cirrhotic and non-cirrhotic chronic liver disease tissue samples show great similarity probably because they are dominated by broad fatty acid signals (Fig. 1). To establish a global overview of the characteristics of the different types of liver tissue studied here, multivariate data analysis was applied to the HR-MAS data. PLS-DA analysis, which is focused on the discrimination of different groups of samples, provided partial separation between cirrhotic and non-cirrhotic samples (Fig. 3A). The Latent Variable 1 (Fig. 3B) showed that the major metabolic differences correspond to the levels of some unsaturated fatty acids, choline, phosphocholine, glucose, glutamate, glutamine, aspartate and phosphoethanolamine. Spectral signal integration was performed over the signals of these metabolites and the significance of the relative integral values with respect to the final diagnosis was assessed. Metabolites with a significantly different signal between cirrhotic and non-cirrhotic tissue according to the MannWhitney $U$ test are reported in Table II and presented in Figs. 4 and 5.

Metabolic profiles. In liver tissue, there is an abundance of large macromolecules, lipids and fatty acids. However, in our study, the liver content in these metabolites shows no clear correlation to the degree of the disease. Variability in the total relative fat content of the different samples, calculated as the added area of the individual fatty acid peaks, seem to have no correlation, at least in the HR-MAS spectra, to the liver disease stage. There were statistically significant differences between cirrhotic and non-cirrhotic liver tissue in the levels of phospholipid intermediates (Table II and Fig. 4). In the case of cirrhotic tissue, the amount of free choline was found to be lower, whereas the amounts of phosphocholine and phosphoethanolamine were found to be higher than the respective amounts in non-cirrhotic tissue. There were also significant variations between cirrhotic and non-cirrhotic samples in some amino acids. In cirrhosis, the levels of glutamate were increased whereas those of glutamine and aspartate were decreased. Further analysis of the levels of glutamate and glutamine also showed some correlation with the disease stage (Glutamate, Pearson correlation coefficient $0.39, \mathrm{p}=0.04$; Glutamine, Pearson correlation coefficient -0.51 , $\mathrm{p}=0.03)$. The mean values for these metabolites in the different disease stages are presented in Fig. 5. Other identified metabolites with statistically significant differences between cirrhotic and non cirrhotic samples include glucose and some unsaturated fatty acids (UFA, signal at $2.70 \mathrm{ppm}$ ). Glucose also showed some correlation (Pearson correlation coefficient $-0.42, p=0.04$ ) with the liver disease stage. 
Table II. The most statistically significant resonance intensity relative values between non-cirrhotic and cirrhotic samples with the standard deviation and the p-values derived from the Mann-Whitney U test.

\begin{tabular}{|c|c|c|}
\hline Metabolite signal/classification & Mean \pm SD & p-value \\
\hline \multicolumn{3}{|l|}{ Unsaturated fatty acyl chains (UFA) } \\
\hline$-\mathrm{CH}=\mathrm{CH}-\mathrm{CH} 2-(2.77 \mathrm{ppm})$ & & 0.062 \\
\hline Chronic hepatitis & $141 \pm 90$ & \\
\hline Cirrhosis & $409 \pm 342$ & \\
\hline Choline (3.20 ppm) & & 0.057 \\
\hline Chronic hepatitis & $134 \pm 43$ & \\
\hline Cirrhosis & $85 \pm 60$ & \\
\hline Phosphocholine (3.22 ppm) & & 0.041 \\
\hline Chronic hepatitis & $148 \pm 67$ & \\
\hline Cirrhosis & $220 \pm 138$ & \\
\hline TMAO (3.26 ppm) & & 0.062 \\
\hline Chronic hepatitis & $244 \pm 02$ & \\
\hline Cirrhosis & $110 \pm 81$ & \\
\hline$\alpha$-glucose (3.41 ppm) & & 0.036 \\
\hline Chronic hepatitis & $91 \pm 31$ & \\
\hline Cirrhosis & $59 \pm 32$ & \\
\hline Glutamate (3.62 ppm) & & 0.031 \\
\hline Chronic hepatitis & $59 \pm 49$ & \\
\hline Cirrhosis & $100 \pm 67$ & \\
\hline Glutamine (3.78 ppm) & & 0.065 \\
\hline Chronic hepatitis & $101 \pm 84$ & \\
\hline Cirrhosis & $27 \pm 69$ & \\
\hline Aspartate (3.86 ppm) & & 0.049 \\
\hline Chronic hepatitis & $83 \pm 27$ & \\
\hline Cirrhosis & $51 \pm 82$ & \\
\hline B-glucose (3.89 ppm) & & 0.067 \\
\hline Chronic hepatitis & $119 \pm 133$ & \\
\hline Cirrhosis & $60 \pm 23$ & \\
\hline Phosphoethanolamine (4.01 ppm) & & 0.044 \\
\hline Chronic hepatitis & $290 \pm 131$ & \\
\hline Cirrhosis & $497 \pm 302$ & \\
\hline
\end{tabular}

\section{Discussion}

Metabolic profiles of chronic liver disease biopsies provided differential patterns between cirrhosis and non-cirrhosis and allow the determination of progressive metabolic alterations associated to chronic hepatic disease. Previous studies of similar characteristics focused their analysis and conclusions in the study of lipid composition (21). In this work, we report that metabolic alterations associated to liver disease stage affect essential metabolic processes beyond lipid metabolism. Early stages of chronic liver disease seem to have important metabolic consequences.

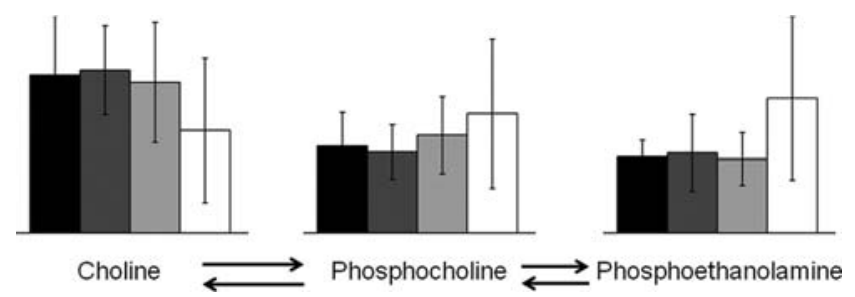

Figure 4. Scheme showing bar graphs for the metabolites involved in phospholipid metabolism at the different liver disease stages (1, black; 2, dark gray; 3 , light gray; 4/cirrhosis, white).

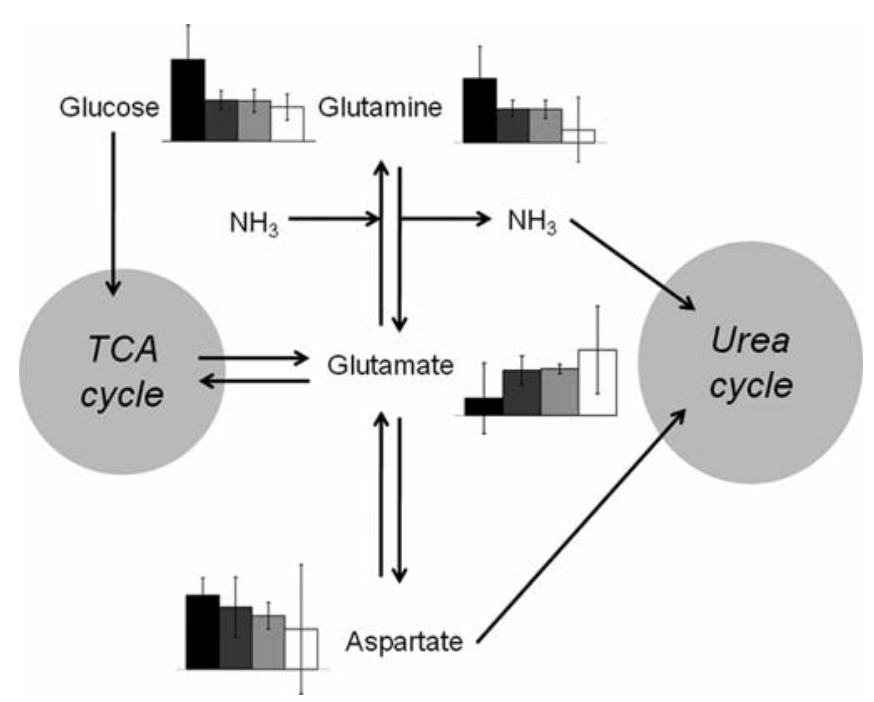

Figure 5. Scheme showing bar graphs for the metabolites involved in the ammonia detoxification by the liver at the different liver disease stages (1, black; 2, dark gray; 3 , light gray; 4/cirrhosis, white).

Choline-containing compounds are important molecules in the synthesis and degradation of phospholipids, which are an essential component of the different membranes. We observed some changes in the levels of these molecules in liver cirrhosis. Similar alterations in the levels of phospholipid precursors have been reported both 'in vitro' and 'in vivo' in previous studies $(42,43)$. The interpretation of variations in these signals is not trivial. Altered intensity of these signals may not only suggest altered synthesis or degradation of membranes, but it may also indicate changes in the synthesis and the degradation combined or just in the mobility of the choline-containing compound parts. In the case of liver fibrosis, which is characterized by the replacement of liver tissue by fibrous scar tissue, the decrease in choline may reflect a decrease in membrane metabolism and possibly in liver functional tissue and hepatocytes. Phosphoethanolamine, which is also an intermediate in the metabolism of phospholipids, is increased in cirrhotic tissue. However, its detailed physiological significance is largely unknown. Differently to choline-containing compounds, phosphoethanolamine does not appear as a common intermediate in membrane degradation. Increased phosphoethanolamine levels suggest that phosphatidylethanolamine metabolism has been activated or that incorporation of phosphatidylethanolamine in the membranes has been partially impaired. In the case of 
liver disease, the second pathway seems more probable. The methylation of phosphatidylethanolamine, which is stimulated by methionine, is responsible for $30 \%$ of the phosphatidylcholine synthesis in the liver (44). Methionine metabolism is typically altered in advanced liver disease (45), being a probable reason responsible for the abnormal levels of phosphoethanolamine observed in this work. Nevertheless, the metabolic alterations generally observed in advanced liver disease are so complex that many different pathways are probably affected. In fact, levels of TMAO are also decreased, in agreement with previous studies (46), suggesting also a possible impairment of the N-oxidation pathway of choline and glycerophosphorylcholine (47).

Amino acid metabolism, a major hepatic metabolic process, is shifted towards the production and accumulation of glutamate. Most amino acids were easily observable in the spectra of samples with lower relative fat content. Some of them presented interesting differences between cirrhotic and non cirrhotic tissue. Previous HR-MAS studies on human liver biopsies reported decreased alanine in cirrhotic tissue (28). In the spectra presented here, alanine was not visible in all the cases. When alanine was visible, a trend towards decreased levels in cirrhotic tissue was observed although no statistically significant differences were detected. Other amino acids with variations present in human liver diseased tissue include aspartate, glutamine and glutamate. Some of the signals belonging to these molecules are located in regions with no lipid or fatty acid overlapping and, therefore, were easily identified. However, strong differences in the signal-to-noise ratios for these signals due to the variability in samples size and fat content made the quantification quite challenging. Interestingly, glutamine levels in cirrhotic tissue were always lower than those in non cirrhotic tissue samples. The levels of glutamate and glutamine in stages 2 and 3 were very similar (Fig. 5). Glutamine is metabolically linked to the ammonium detoxification in the body by the hepatocytes. Decreased levels of glutamine may reflect increased hepatic dysfunction and/or destruction of hepatocytes. Interestingly, glutamate, which is the precursor of glutamine in the glutamine-synthase reaction, is increased in cirrhotic liver, supporting this conclusion. The lower levels of aspartate and glutamine together with the higher level of glutamate may indicate a shift towards glutamate production, a net release of ammonium and impaired ammonium detoxification ability. Diseased liver is subject to oxidative stress (14). In this context, it is very possible to have a metabolic shift towards the synthesis of precursors of glutathione, a well known cell antioxidant. All these facts are consistent with the hypothesis that increased circulating ammonium is the major culprit for the origin of hepatic encephalopathy (48), a serious complication of advanced liver disease.

The observed decrease in the glucose signal intensity suggests alterations in the glycolysis and in the glucose production in the liver, as has been reported in acute liver injury $(46,47)$. Interestingly, these alterations seem to appear from the early stages of the liver disease (Fig. 4). The transition to cirrhotic liver seems to produce a less dramatic change in the levels of glucose. On the other hand, an increase in the levels of UFAs with the disease stage was detected, albeit with low statistical significance, suggesting that lipid metabolism, an important part of the liver metabolism, is also compromised with the fibrotic stage.

In summary, the metabolic characterization of liver tissue from needle biopsies by HR-MAS NMR spectroscopy provided detailed patterns for cirrhotic and non-cirrhotic chronic liver disease tissue. The metabolic composition of the liver tissue was highly consistent with previous studies. Clear metabolic differences between cirrhosis and noncirrhotic tissue exist. The most relevant of these differences are the glutamate increase and the glutamine and glucose decreases. Other differences include aspartate, choline, phosphocholine and phosphoethanolamine. Correlations with respect to the liver disease stage of some of these metabolites, namely, glutamate, glutamine and glucose, suggest that hepatic function is progressively impaired with the development of hepatic fibrosis. Overall, this work suggests that the additional information obtained by NMR metabolomics applied to needle biopsies of human liver may be useful for assessing metabolic alterations and liver dysfunction in chronic liver disease.

\section{Acknowledgements}

This study was partly financed by grants from the Ministerio de Educación y Ciencia del Gobierno de España (SAF20076547 and SAF2008-00270) and the Consellería de Sanidad de la Generalitat Valenciana (GVASAN/AP014/2009). D.M. gratefully acknowledges the Ministerio de Educación y Ciencia del Gobierno de España for a Ramon y Cajal 2006 Contract.

\section{References}

1. Guyton AC and Hall JE (eds.): Medical Physiology. Elsevier Saunders, Philadelphia, PA, 2006.

2. Ros PR and Mortele KJ: Diffuse liver disease. Clin Liver Dis 6: 181-201, 2002.

3. Simpson KJ and Finlayson ND: Clinical evaluation of liver disease. Baillieres Clin Gastroenterol 9: 639-659, 1995.

4. Friedman LS and Keeffe B (eds.): Handbook of liver disease. Churchill Livingstone, London, 2004.

5. Hunt $\mathrm{N}$ and Fleming K: Reproducibility of liver biopsy grading and staging. Liver 19: 169-170, 1999.

6. Brunt EM: Grading and staging the histopathological lesions of chronic hepatitis: the Knodell histology activity index and beyond. Hepatology 31: 241-246, 2000.

7. Ishak K, Baptista A, Bianchi L, et al: Histological grading and staging of chronic hepatitis. J Hepatol 22: 696-699, 1995.

8. Friedman SL: Liver fibrosis - from bench to bedside. J Hepatol 38 (Suppl 1): S38-S53, 2003.

9. Pinzani M, Rombouts $\mathrm{K}$ and Colagrande S: Fibrosis in chronic liver diseases: diagnosis and management. J Hepatol 42: S22-S36, 2005.

10. Dezortova M, Taimr P, Skoch A, Spicak J and Hajek M: Etiology and functional status of liver cirrhosis by 31P MR spectroscopy. World J Gastroenterol 11: 6926-6931, 2005.

11. Kuriyama S, Yokoyama F, Inoue H, et al: Sequential assessment of the intrahepatic expression of epidermal growth factor and transforming growth factor-beta1 in hepatofibrogenesis of a rat cirrhosis model. Int J Mol Med 19: 317-324, 2007.

12. Canbay A, Friedman S and Gores GJ: Apoptosis: the nexus of liver injury and fibrosis. Hepatology 39: 273-278, 2004.

13. Ramadori G and Saile B: Inflammation, damage repair, immune cells, and liver fibrosis: specific or nonspecific, this is the question. Gastroenterology 127: 997-1000, 2004.

14. Poli G: Pathogenesis of liver fibrosis: role of oxidative stress. Mol Aspects Med 21: 49-98, 2000.

15. Gutierrez-Reyes G, Gutierrez-Ruiz MC and Kershenobich D: Liver fibrosis and chronic viral hepatitis. Arch Med Res 38: 644-651, 2007. 
16. Kaji K, Yoshiji H, Kitade M, et al: Impact of insulin resistance on the progression of chronic liver diseases. Int J Mol Med 22: 801-808, 2008 .

17. Naveau S, Raynard B, Ratziu V, et al: Biomarkers for the prediction of liver fibrosis in patients with chronic alcoholic liver disease. Clin Gastroenterol Hepatol 3: 167-174, 2005.

18. Ratziu V, Giral P, Munteanu M, et al: Screening for liver disease using non-invasive biomarkers (FibroTest, SteatoTest and NashTest) in patients with hyperlipidaemia. Aliment Pharmacol Ther 25: 207-218, 2007.

19. Salcedo X, Medina J, Sanz-Cameno P, Garcia-Buey L, MartinVilchez S and Moreno-Otero R: Review article: angiogenesis soluble factors as liver disease markers. Aliment Pharmacol Ther 22: 23-30, 2005.

20. Parola M, Robino G and Dianzani MU: 4-hydroxy-2,3-alkenals as molecular mediators of oxidative stress in the pathogenesis of liver fibrosis (review). Int J Mol Med 4: 425-432, 1999.

21. Cobbold JF, Patel JH, Goldin RD, et al: Hepatic lipid profiling in chronic hepatitis $\mathrm{C}$ : An in vitro and in vivo proton magnetic resonance spectroscopy study. J Hepatol 52: 16-24, 2010.

22. Fischbach $\mathrm{F}$ and Bruhn $\mathrm{H}$ : Assessment of in vivo $1 \mathrm{H}$ magnetic resonance spectroscopy in the liver: a review. Liver Int 28 : 297-307, 2008.

23. Sijens PE, Edens MA, Bakker SJ and Stolk RP: MRI-determined fat content of human liver, pancreas and kidney. World J Gastroenterol 16: 1993-1998, 2010.

24. Springer F, Machann J, Claussen CD, Schick F and Schwenzer NF: Liver fat content determined by magnetic resonance imaging and spectroscopy. World J Gastroenterol 16: 1560-1566, 2010

25. Cheng LL, Ma MJ, Becerra L, et al: Quantitative neuropathology by high resolution magic angle spinning proton magnetic resonance spectroscopy. Proc Natl Acad Sci USA 94: 6408-6413, 1997.

26. Sitter B, Bathen TF, Singstad TE, et al: Quantification of metabolites in breast cancer patients with different clinical prognosis using HR MAS MR spectroscopy. NMR Biomed: January 25, 2010 (Epub ahead of print).

27. Martinez-Bisbal MC, Monleon D, Assemat O, et al: Determination of metabolite concentrations in human brain tumour biopsy samples using HR-MAS and ERETIC measurements. NMR Biomed 22: 199-206, 2009.

28. Martinez-Granados B, Monleon D, Martinez-Bisbal MC, et al Metabolite identification in human liver needle biopsies by high-resolution magic angle spinning $1 \mathrm{H}$ NMR spectroscopy. NMR Biomed 19: 90-100, 2006.

29. Monleon D, Morales JM, Gonzalez-Darder J, et al: Benign and atypical meningioma metabolic signatures by high-resolution magic-angle spinning molecular profiling. J Proteome Res 7 : 2882-2888, 2008.

30. Griffin JL, Bollard M, Nicholson JK and Bhakoo K: Spectral profiles of cultured neuronal and glial cells derived from HRMAS (1)H NMR spectroscopy. NMR Biomed 15: 375-384, 2002.

31. Hong YS, Coen M, Rhode CM, et al: Chemical shift calibration of $1 \mathrm{H}$ MAS NMR liver tissue spectra exemplified using a study of glycine protection of galactosamine toxicity. Magn Reson Chem 47 (Suppl 1): S47-S53, 2009.

32. Swanson MG, Zektzer AS, Tabatabai ZL, et al: Quantitative analysis of prostate metabolites using $1 \mathrm{H}$ HR-MAS spectroscopy. Magn Reson Med 55: 1257-1264, 2006.
33. Weybright P, Millis K, Campbell N, Cory DG and Singer S: Gradient, high-resolution, magic angle spinning $1 \mathrm{H}$ nuclear magnetic resonance spectroscopy of intact cells. Magn Reson Med 39: 337-345, 1998.

34. Cheng LL, Chang IW, Louis DN and Gonzalez RG: Correlation of high-resolution magic angle spinning proton magnetic resonance spectroscopy with histopathology of intact human brain tumor specimens. Cancer Res 58: 1825-1832, 1998

35. Maxwell RJ, Martinez-Perez I, Cerdan S, et al: Pattern recognition analysis of $1 \mathrm{H}$ NMR spectra from perchloric acid extracts of human brain tumor biopsies. Magn Reson Med 39: 869-877, 1998 .

36. Duarte IF, Stanley EG, Holmes E, et al: Metabolic assessment of human liver transplants from biopsy samples at the donor and recipient stages using high-resolution magic angle spinning $1 \mathrm{H}$ NMR spectroscopy. Anal Chem 77: 5570-5578, 2005.

37. Cox IJ, Sharif A, Cobbold JF, Thomas HC and TaylorRobinson SD: Current and future applications of in vitro magnetic resonance spectroscopy in hepatobiliary disease. World J Gastroenterol 12: 4773-4783, 2006.

38. Martinez-Bisbal MC, Marti-Bonmati L, Piquer J, et al: $1 \mathrm{H}$ and 13C HR-MAS spectroscopy of intact biopsy samples ex vivo and in vivo $1 \mathrm{H}$ MRS study of human high grade gliomas. NMR Biomed 17: 191-205, 2004.

39. Ludwig J, Batts KP, Moyer TP and Poterucha JJ: Advances in liver biopsy diagnosis. Mayo Clin Proc 69: 677-678, 1994.

40. Waters NJ, Garrod S, Farrant RD, et al.: High-resolution magic angle spinning (1)H NMR spectroscopy of intact liver and kidney: optimization of sample preparation procedures and biochemical stability of tissue during spectral acquisition. Anal Biochem 282: 16-23, 2000.

41. Piotto M, Elbayed K, Wieruszeski JM and Lippens G: Practical aspects of shimming a high resolution magic angle spinning probe. J Magn Reson 173: 84-89, 2005.

42. Schlemmer HP, Sawatzki T, Sammet S, et al: Hepatic phospholipids in alcoholic liver disease assessed by protondecoupled 31P magnetic resonance spectroscopy. J Hepatol 42: 752-759, 2005

43. Tarachiwin L, Ute K, Kobayashi A and Fukusaki E: $1 \mathrm{H}$ NMR based metabolic profiling in the evaluation of Japanese green tea quality. J Agric Food Chem 55: 9330-9336, 2007.

44. Sundler R and Akesson B: Regulation of phospholipid biosynthesis in isolated rat hepatocytes. Effect of different substrates. J Biol Chem 250: 3359-3367, 1975

45. Mato JM and Ortiz P: Metabolism of methionine in liver disease. Methods Find Exp Clin Pharmacol 18 (Suppl B): 35-46, 1996.

46. Constantinou MA, Tsantili-Kakoulidou A, Andreadou I, Iliodromitis EK, Kremastinos DT and Mikros E: Application of NMR-based metabonomics in the investigation of myocardial ischemia-reperfusion, ischemic preconditioning and antioxidant intervention in rabbits. Eur J Pharm Sci 30: 303-314, 2007.

47. Waters NJ, Waterfield CJ, Farrant RD, Holmes E and Nicholson JK: Metabonomic deconvolution of embedded toxicity: application to thioacetamide hepato- and nephrotoxicity. Chem Res Toxicol 18: 639-654, 2005.

48. Rodrigo R, Cauli O, Gomez-Pinedo U, et al: Hyperammonemia Induces Neuroinflammation that Contributes to Cognitive Impairment in Rats with Hepatic Encephalopathy. Gastroenterology 139: 675-684, 2010. 